\title{
Noninvasive Brain Stimulation in Stroke Rehabilitation
}

\author{
Brian R. Webster, Pablo A. Celnik, and Leonardo G. Cohen \\ Human Cortical Physiology Section and Stroke Neurorehabilitation Clinic, National Institute of Neurological Disorders and \\ Stroke, National Institutes of Health, Bethesda, Maryland 20892
}

\begin{abstract}
Summary: Stroke is a common disorder that produces a major burden to society, largely through long-lasting motor disability in survivors. Recent studies have broadened our understanding of the processes underlying recovery of motor function after stroke. Bilateral motor regions of the brain experience substantial reorganization after stroke, including changes in the strength of interhemispheric inhibitory interactions. Our understanding of the extent to which different forms of reorganization contribute to behavioral gains in the rehabilitative process, although still limited, has led to the formulation of novel interventional strategies to regain motor function. Transcranial magnetic (TMS) and DC (tDCS) electrical stimulation are noninvasive brain stimulation tech-
\end{abstract}

niques that modulate cortical excitability in both healthy individuals and stroke patients. These techniques can enhance the effect of training on performance of various motor tasks, including those that mimic activities of daily living. This review looks at the effects of TMS and tDCS on motor cortical function and motor performance in healthy volunteers and in patients with stroke. Both techniques can either enhance or suppress cortical excitability, and may move to the clinical arena as strategies to enhance the beneficial effects of customarily used neurorehabilitative treatments after stroke. Key Words: Stroke, motor cortex, rehabilitation, cortical stimulation, transcranial magnetic stimulation (TMS), transcranial direct current stimulation (tDCS), plasticity.

\section{INTRODUCTION}

An estimated 700,000 Americans suffered a stroke during 2005, incurring estimated costs related to their care of approximately $\$ 56.8$ billion. ${ }^{1}$ Stroke is a leading cause of serious long-term disability, and approximately 1.1 million Americans with stroke had functional limitations in activities of daily living in $1999 .^{2}$ The burden of stroke-related disability is predicted to increase in the coming decades in proportion to the expansion of the elderly population. ${ }^{3}$ Stroke case-fatality has declined, but stroke incidence has not, leading to rising numbers of stroke survivors.

After ischemic damage to motor areas of the brain, patients experience some degree of spontaneous recovery, ${ }^{4,5}$ increasingly so since the advent of interventions implemented in the acute period after stroke-notably, use of tissue plasminogen activator (TPA) to dissolve blood clots. TPA represents an important advance in the fight against this disease, but so far has benefited a limited proportion of stroke patients. ${ }^{6}$ More than $50 \%$ of stroke

Address correspondence and reprint requests to: Leonardo G. Cohen, M.D., Human Cortical Physiology Section, National Institute of Neurological Disorders and Stroke, National Institutes of Health, Bethesda, MD 20817. E-mail: cohen1@ninds.nih.gov. survivors who reach the chronic stage experience permanent motor deficits. ${ }^{7}$ Repetitive task-oriented motor training represents the current standard in neurorehabilitation after chronic stroke. ${ }^{8,9}$ In recent years, new strategies in repetitive motor training have raised substantial interest, including constraint-induced therapy, ${ }^{10-12}$ bilateral arm training, ${ }^{13,14}$ body-weight support treadmill training, ${ }^{15-17}$ robotic assisted therapy, ${ }^{18-24}$ and use of virtual reality protocols. ${ }^{25-30}$

\section{MECHANISMS OF FUNCTIONAL RECOVERY}

Studies of cortical plasticity after stroke suggest that the damaged cortex has the potential for extensive reorganization (for a review, see Ward and Cohen ${ }^{31}$ and Calautti and Baron $^{32}$ ). Among possible mechanisms of neural plasticity contributing to functional recovery are dendritic sprouting over time, ${ }^{33,34}$ new synapse formation, ${ }^{35}$ and long-term potentiation (LTP) and depression (LTD). ${ }^{36,37}$ Reorganization after stroke may also involve undamaged areas of cortex taking on functions of the infarcted regions. ${ }^{38}$ Different forms of reorganization that may contribute to functional recovery include diaschisis, peri-infarct reorganization, activity in the ipsile- 
sional or in the contralesional hemisphere, interhemispheric interactions, and vicarious reorganization.

\section{Diaschisis}

In diaschisis, there is functional deactivation of undamaged areas of the CNS that are remote but connected to the lesioned area. ${ }^{39}$ Imaging studies in stroke patients have shown diaschisis in cerebellar structures in the motor domain ${ }^{40-44}$ and also in other regions related to language ${ }^{45}$ and vision ${ }^{46}$ processing. Resolution of cerebellar diaschisis has been proposed as one of the mechanisms contributing to recovery of motor function after stroke ${ }^{47,48}$ for example, after intake of amphetamines. ${ }^{49,50}$

\section{Peri-infarct reorganization}

Peri-infarct reorganization can occur after focal cortical lesions in the primary motor cortex. ${ }^{51,52}$ This form of plasticity, described in nonhuman primates, may contribute to motor recovery by allowing other areas near the lesioned area to gain control of the weak body part. ${ }^{51,52}$ In humans, Jaillard et al ${ }^{53}$ for 2 years followed a group of four stroke patients who had a small lesion located in the primary motor cortex (M1) and found that recovery of finger movements was associated with a dorsal shift of the cortical activation areas within M1. Note, however, that such focal cortical lesions restricted to M1 are not commonly seen in stroke patients. Additionally, in human studies the increased magnitude of peri-infarct activation seen under functional MRI (fMRI) ${ }^{54}$ does not show a direct correlation with the magnitude of motor recovery, ${ }^{55}$ raising the question of the degree to which peri-infarct reorganization contributes directly to motor recovery. Such findings underscore both the importance of designing realistic animal models of human stroke, and its complexity.

\section{Activity in the ipsilesional hemisphere}

In addition to the peri-infarct rim, increasing levels of activation have been reported in a distributed ipsilesional network that includes primary motor cortex, premotor cortex, supplementary motor area, and bilateral Brodmann area 40 in stroke. These findings correlated positively with one index of motor recovery, the hand score of the motricity index. ${ }^{56}$ Taken together, these neuroimaging studies suggest that activation in ipsilesional motor areas plays an important role in the recovery process. Neurophysiological studies showed that transient disruption of activity in the ipsilesional M1 and dorsal premotor cortex of patients with chronic stroke and good motor recovery caused clear transient deficits in motor performance of the paretic hand. ${ }^{57,58}$ Altogether, these findings suggest that motor performance in the paretic hand of patients with good motor recovery relies predominantly on reorganized activity within the lesioned hemisphere. ${ }^{31}$

\section{Activity in the contralesional hemisphere}

The unaffected hemisphere is another important locus of reorganization after stroke. Increased levels of contralesional sensorimotor activation have been identified during simple movements of the paretic hand. ${ }^{59,60}$ The magnitude of contralesional activation appears to decrease in M1 at 3 to 6 months, relative to 1 week after the stroke, ${ }^{60,32}$ but the intensity of contralesional M1 activity does not correlate with the degree of recovery. ${ }^{56}$ This finding might indicate that contralesional activation is not functionally relevant for recovery, ${ }^{56}$ or that it is insufficient to compensate for a marked motor deficit.

Increased contralesional activation may be most prominent in the early stages after stroke, and with later refocusing of activation back to the ipsilesional motor regions. ${ }^{61}$ Consistent with this view, disruption of activity in contralesional M1 did not greatly disrupt paretic hand function in patients with chronic subcortical stroke and good motor recovery. ${ }^{57}$ On the other hand, disruption of activity in the contralesional dorsal premotor cortex resulted in disruption of a reaction-time task in patients with poorer recovery. ${ }^{62}$ Moreover, higher levels of contralesional activity in secondary motor areas (prefrontal and parietal cortices) appear to be predictive of a slower motor recovery, suggesting a possible role for activity in the intact hemisphere in patients with lesser or unsuccessful recovery. ${ }^{56} \mathrm{~A}$ recent study, however, applied fMRI-guided TMS overactivated areas of the contralesional hemisphere and found that a complex behavioral task could be disrupted in chronic subcortical stroke patients but not in a healthy age-matched group. ${ }^{63}$

Regardless of the specific role of each hemisphere, it is clear that recovery processes are likely to rely on the operation of more extensive ensembles of neurons organized in neural networks that link activity in different cortical sites of both hemispheres. Additionally, the role that activity in the intact and affected hemispheres plays in motor control is likely to vary, depending on lesion sites, time from stroke, complexity of the behavioral task, and magnitude of impairment. ${ }^{31,63,64}$ If this is the case, caution is advised in drawing definite conclusions about the involvement of different ipsi- and contralesional regions in the process of functional recovery.

\section{Interhemispheric interactions}

Interactions between the hemispheres can also contribute to reorganization. One example is interhemispheric inhibition between homonymous motor cortical representations. ${ }^{65}$ Transcallosal fibers are known to transmit inhibitory influences between the homologous areas of both hemispheres. ${ }^{66}$ These fibers are thought to be glutamatergic and to project onto inhibitory GABAergic interneurons. ${ }^{67}$ Patients with stroke have changes in motor cortical excitability ${ }^{68-70}$ and an abnormally high in- 
terhemispheric inhibition from the contralesional M1 to the ipsilesional M1 with movements of the paretic hand.

These changes are observed in association with movements of the paretic hand, but less so or not at all at rest. The increased interhemispheric inhibition is more prominent in cases with more substantial motor impairment, ${ }^{65}$ a finding consistent with previous work on interhemispheric competition in sensory areas. These findings led to the proposal of a similar model in the motor domain (see Ward and Cohen ${ }^{31}$ ). Under this model, purposeful modulation of excitability in motor regions of the intact and affected hemisphere using noninvasive brain stimulation could potentially contribute to functional restoration. $^{71}$

\section{Vicarious reorganization}

In vicarious reorganization, a healthy region of the brain could take over the function of another, lesioned brain area. The term has been used to explain the finding of functional recovery after certain brain lesions. ${ }^{72}$ Evidence for this type of process exists in animal models ${ }^{51,73,74}$ and in humans. ${ }^{53}$

\section{NONINVASIVE BRAIN STIMULATION}

Recent work has raised the possibility that brain stimulation can enhance the beneficial effects of motor training in the rehabilitative process. ${ }^{31}$ Studies in animal models showed that motor recovery after focal lesions in the primary motor cortex can improve with direct epidural cortical stimulation. ${ }^{75,76}$ Human studies addressing this possibility are currently underway. ${ }^{75}$ In parallel with these invasive approaches, there has been increasing interest in testing the effects of noninvasive cortical stimulation in the rehabilitative process. Transcranial magnetic stimulation (TMS) and transcranial direct current stimulation (tDCS) have been investigated as potential tools for modulating motor recovery in stroke or influencing motor, sensory, and cognitive functions.

TMS is delivered by passing a strong brief electrical current through an insulated coil placed on the skull. The current induces a transient magnetic field in the brain, and electric currents in the cortex flow parallel to the coil, thereby depolarizing neurons. TMS can enhance or decrease activity in cortical regions and influence function, depending on multiple variables such as the frequency, duration of stimulation, the shape of the coil, and the strength of the magnetic field. The effects of repetitive TMS (rTMS) on cortical excitability can outlast the stimulation period for as much as 1 to 2 hours. ${ }^{77,78}$

tDCS at intensities of 1 to $2 \mathrm{~mA}$ is applied through two surface electrodes placed on the skull. Depending on the duration and the polarity of the stimulation, tDCS can increase or depress excitability in the stimulated region from minutes to 1 to 2 hours. tDCS does not appear to induce direct neuronal depolarization, as does TMS; instead, it modulates the activation of sodium- and calcium-dependent channels and NMDA receptor activity, promoting LTP- and LTD-like changes. ${ }^{79-81}$

The duration of effects elicited by a single application of either TMS or tDCS appears to be comparable (up to hours). Both techniques are noninvasive and appear to be safe when used within established safety guidelines. The mechanisms underlying their effects, however, may differ. TMS equipment is more expensive, but can stimulate more focally and exhibits a better time resolution (TMS can be applied with millisecond accuracy, but tDCS requires several minutes). There is more experience in the use of TMS protocols than of tDCS, but for double-blind experimental designs sham tDCS is easier to achieve than sham TMS. ${ }^{82}$ In rehabilitative settings, tDCS is more easily applied simultaneously with cognitive or motor training protocols. Thus, the choice of technique depends largely on the particular hypothesis to be tested and the experimental setting.

In terms of safety, the main issue raised with rTMS has been the possibility of seizures; however, available rules of application and training protocols have made them a rare event. ${ }^{83}$ Individuals with history of seizures are largely excluded from rTMS studies (except in trials geared to test the possibility of beneficial effects in some forms of epilepsy). For tDCS, on the other hand, there are no reports of seizures, but safety studies are still required. tDCS often elicits short-lasting tingling sensations at the beginning and end of the stimulation period, rarely accompanied by redness under the electrode sites. ${ }^{81,82,84}$ Occasional transient headaches have been described for both TMS and tDCS. Both rTMS and tDCS appear to be safe if used within proposed safety limits.

Additional points to keep in mind are that: (1) application of TMS or tDCS to one cortical site will probably influence distant cortical or subcortical sites through trans-synaptic effects ${ }^{85,86}$; (2) in patients with brain lesions, expected models of current flow elicited by rTMS or tDCS may differ from those in healthy volunteers ${ }^{87}$; and (3) both techniques may potentially influence attention, fatigue, discomfort, or mood, which underscores the importance of controlling for these factors in the design of double-blind clinical trials.

Recent work has documented beneficial effects of noninvasive brain stimulation on cognitive functions in healthy humans and nonhuman primates. High-frequency rTMS applied to the primary motor cortex resulted in performance improvements in reaction-time $\operatorname{tasks}^{88}$ and in motor sequence learning. ${ }^{89}$ Consistent with these data, application to primary somatosensory cortex produced lasting improvements in two-point tactile discrimination and an enlargement of the right index finger representation in $\mathrm{S} 1$, as measured by fMRI. ${ }^{90}$

Similar findings have been reported with tDCS. Early 


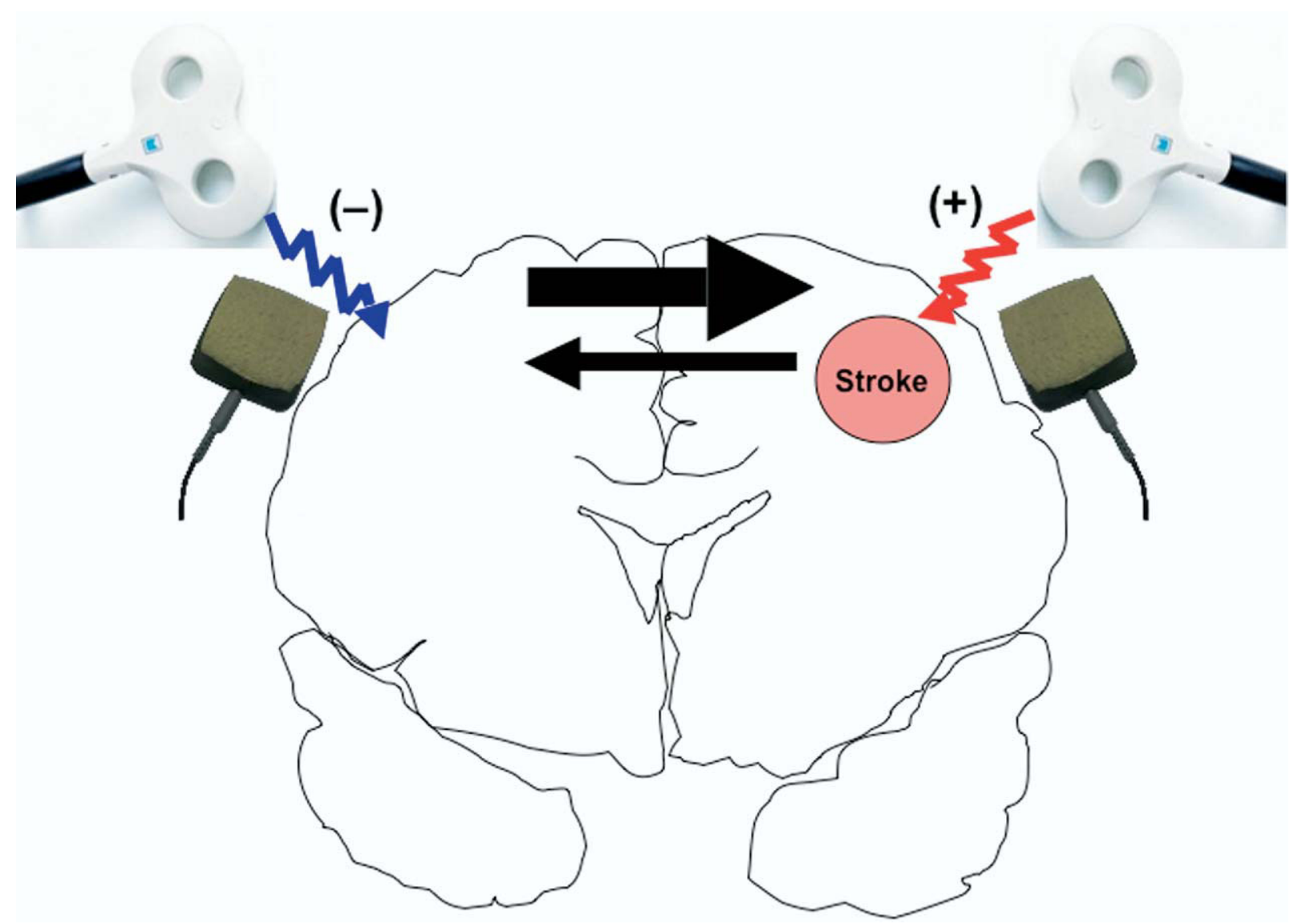

FIG. 1. Possible therapeutic uses of noninvasive brain stimulation to promote recovery in chronic stroke (in this diagram, transcranial magnetic stimulation [TMS] and transcranial direct current stimulation [tDCS]). Cortical reorganization after stroke produces an imbalance of interhemispheric inhibition, which may be reduced by inhibitory TMS or transcranial direct current stimulation (tDCS) to the contralesional hemisphere. Alternatively, beneficial forms of reorganization may be promoted by excitatory TMS or tDCS to the ipsilesional hemisphere.

studies in nonhuman primates demonstrated that anodal tDCS to dorsolateral prefrontal (DLPF) cortex could improve learning of a simple motor reaction time task. ${ }^{91}$ In humans, ${ }^{92,93}$ application of anodal tDCS has been reported to improve implicit learning when applied to the primary motor cortex ${ }^{94}$ and to enhance working memory and probabilistic classification learning when applied to the DLPF cortex..$^{95,96}$ Visuomotor learning was facilitated with anodal tDCS applied to the primary motor cortex or to area V5 of occipital cortex..$^{97}$

The mechanisms underlying these effects are incompletely understood, but they could include modulation of LTP- or LTD-like processes, ${ }^{98}$ as well as nonspecific changes such as generalized postsynaptic excitability enhancements. ${ }^{99}$ These well documented effects of noninvasive brain stimulation in healthy subjects support possible application in stroke patients.

Models of interhemispheric competition ${ }^{71}$ suggest possible strategies to increase the beneficial effects of motor training on function in the paretic hand (FIG. 1): enhancing excitability in the ipsilesional M1 and decreasing excitability in the contralesional M1. Neuro- modulation of activity in the dorsal ${ }^{58,62}$ and ventral ${ }^{100}$ premotor cortices or the supplementary motor area are under active investigation. ${ }^{31}$

\section{Enhancing excitability in the ipsilesional motor cortex}

Both tDCS and rTMS have been used to enhance excitability in the ipsilesional primary motor cortex. Anodal tDCS applied to the ipsilesional M1 has been studied in patients with chronic stroke in shamcontrolled double-blind crossover experimental designs. ${ }^{95,101,102}$ In the first double-blind sham controlled study in 6 patients, each with a single ischemic subcortical cerebral infarct, ${ }^{101}$ the authors documented transient improvements in performance of the JebsenTaylor hand-function test (JTT) ${ }^{103}$ with one single session of stimulation but not with sham. This improvement was evident in all patients studied, representing approximately a $10 \%$ reduction in the time required to perform the JTT, and persisted for more than 30 minutes after the end of the stimulation period. The beneficial behavioral effect in this study was as- 
sociated with increased motor cortical excitability and reduced intracortical inhibition in ipsilesional M1. Although no improvement was observed after sham stimulation, there was a trend toward a greater improvement in subtests of the JTT that emphasize fine motor control of the distal hand muscles, such as turning over cards and picking up objects with a spoon, compared with activities related with upper arm motor function. Improvements in the JTT are highly relevant to a stroke patient's ability to perform everyday activities and have been shown to correlate well with functional rehabilitation. ${ }^{104-106}$

In a study using a single session of rTMS in patients with chronic stroke, Kim et al. ${ }^{89}$ showed that high-frequency rTMS $(10 \mathrm{~Hz})$ to the ipsilesional M1 resulted in a significantly larger increase in MEP amplitudes than sham rTMS; this increase was associated with an enhanced accuracy during performance of a finger motor sequence task. In a study performed in subacute instead of chronic stroke patients, with multiple sessions of rTMS applied to the ipsilesional M1, Khedr et al. ${ }^{107}$ used rTMS (10 trains of $3 \mathrm{~Hz}$ stimulation, duration $10 \mathrm{sec}-$ onds, with 50 seconds between each train, twice daily) combined with customary rehabilitative treatment for 10 days within the first 2 weeks after stroke. They reported performance improvements with rTMS relative to sham lasting for at least 10 days after the end of the treatment period. ${ }^{107}$ None of the studies that stimulated the ipsilesional primary motor cortex reported complications other than transient headache.

Such studies have led to the proposal that repetitive stimulating sessions could elicit longer lasting effects than single applications, as well as the idea that association of cortical stimulation with motor training could enhance the benefit.

\section{Decreasing excitability in the contralesional motor cortex}

Based on the idea that interhemispheric interactions can influence motor performance, ${ }^{108}$ it should be possible, in theory, to improve motor function in the paretic hand by decreasing excitability in the contralesional M1-possibly through modulation of inappropriate interhemispheric inhibition. ${ }^{65}$ Studies in normal volunteers showed that decreasing excitability in one M1 results in increased excitability in the opposite M1 $1^{109,110}$ and even in performance improvements in motor function of the ipsilateral hand. ${ }^{111}$ In patients with stroke, it was shown that cathodal tDCS applied to the contralesional M1 may improve performance in the paretic hand, possibly by suppressing the imbalance in interhemispheric inhibition proposed to interfere with stroke recovery in some patients. ${ }^{95}$ Decreasing activity in the contralesional M1 with $1 \mathrm{~Hz}$ rTMS was recently found to decrease interhemispheric inhibition from the contralesional to ipsile- sional hemisphere of chronic stroke patients ${ }^{112}$; the decrease in interhemispheric inhibition elicited by rTMS correlated with functional improvements in a finger pinch acceleration task. Another study, using low-frequency rTMS to the contralesional motor cortex, found behavioral improvements in the paretic hand in simple reaction time and on the Purdue Pegboard test. ${ }^{113}$ In a study of 6 chronic stroke patients by Fregni et al., ${ }^{95}$ the effects of cathodal tDCS applied to the contralesional primary motor cortex was compared with the effects of anodal stimulation to the affected hemisphere and sham stimulation, again using the JTT time as the behavioral outcome measure. Their results for anodal tDCS to the affected hemisphere ${ }^{95}$ were consistent with those of Hummel et al. ${ }^{101}$ (Note, however, that the patient population studied by Fregni et al. ${ }^{95}$ was subacute stroke patients more severely affected than those studied by Hummel et al., ${ }^{101}$ limiting to some extent the comparability of the 2 studies.) In that study by Fregni et al., ${ }^{95}$ cathodal stimulation to the contralesional M1 produced a significant improvement of $11.7 \%$ on motor performance relative to sham.

The therapeutic studies conducted in stroke patients thus far have been limited to interventions consisting of a single session of approximately 20 minutes of stimulation. It is likely that longer sessions or multiple applications could lead to longer lasting beneficial effects, as shown by Khedr et al. ${ }^{107}$ using rTMS. Another potential advantage of this approach over stimulation of the ipsilesional M1 is that it is applied to healthy neural structures.

Although the majority of interventional studies of TMS in stroke patients have focused on motor function, some studies have sought to test the ability of decreasing activity in the contralesional hemisphere to improve language function, including nonfluent and global aphasia. ${ }^{114,115}$ Overall, their findings further support the concept that repeated interventions (in this case, to decrease activity in the contralesional hemisphere) can lead to behavioral gains in speech function that remain stable over lengthy periods, possibly through modulation of inhibitory interhemispheric interactions. Interestingly, prior to the TMS intervention in one patient, ${ }^{115}$ speech therapy had been discontinued due to lack of progress and poor prognostic indicators.

\section{PERSPECTIVES}

Studies of noninvasive brain stimulation have shown promising results thus far, but there are some limitations to be kept in mind. The studies were obtained in relatively small and selected groups of subjects, often with moderate remaining motor function. The magnitude of improvements reported so far ranged between $10 \%$ and $30 \%$, and we do not know how long lasting they will 
prove to be. This issue could be addressed in future studies using multiple applications of tDCS or TMS.

It would be useful to determine the best parameters and type of experimental sham controls required to optimize and detect the effects. Similarly, we need to better understand the influence of lesion site, chronicity, and levels of impairment on the magnitude of the response to noninvasive stimulation, as well as on the duration of effects. More important, the behavioral endpoint measures in these studies have differed: some ecologically valid, others less so. In addition to this problem, it is conceivable that performance of different tasks engages activity in different neural networks after stroke, making comparisons across studies even more difficult.

In conclusion, TMS and tDCS are emerging as powerful tools for modulating brain activity and cognitive function in stroke patients, with the goal of contributing to rehabilitation. Larger scale double-blind sham-controlled clinical trials are needed to determine the validity of these novel interventions.

Acknowledgments: This work was supported by the Intramural Research Program of the National Institute of Neurological Disorders and Stroke, National Institutes of Health, Bethesda, MD. We thank Dr. Isabelle Loubinoux for helpful comments on the advanced version of this article.

\section{REFERENCES}

1. American Heart Association Writing Group for the Statistics Committee and Stroke Statistics Subcommittee. Heart disease and stroke statistics: 2005 update. Dallas, Texas: AHA, 2005.

2. Centers for Disease Control and Prevention. Prevalence of disabilities and associated health conditions among adults: United States, 1999. MMWR Morb Mortal Wkly Rep 2001;50:120-5. [Erratum in: MMWR Morb Mortal Wkly Rep 2001;50:149]

3. Kavanagh S, Knapp M, Patel A. Costs and disability among stroke patients. J Public Health Med 1999;21:385-394.

4. Wade DT, Langton-Hewer R, Wood VA, Skilbeck CE, Ismail HM. The hemiplegic arm after stroke: measurement and recovery. J Neurol Neurosurg Psychiatry 1983;46:521-524.

5. Sunderland A, Tinson D, Bradley L, Hewer RL. Arm function after stroke: an evaluation of grip strength as a measure of recovery and a prognostic indicator. J Neurol Neurosurg Psychiatry 1989;52:1267-1272.

6. Demaerschalk BM, Yip TR. Economic benefit of increasing utilization of intravenous tissue plasminogen activator for acute ischemic stroke in the United States. Stroke 2005;36:2500-2503.

7. Duncan PW, Goldstein LB, Matchar D, Divine GW, Feussner J. Measurement of motor recovery after stroke: outcome assessment and sample size requirements. Stroke 1992;23:1084-1089.

8. Barreca S, Wolf SL, Fasoli S, Bohannon R. Treatment interventions for the paretic upper limb of stroke survivors: a critical review. Neurorehabil Neural Repair 2003;17:220-226.

9. Krakauer JW. Motor learning: its relevance to stroke recovery and neurorehabilitation. Curr Opin Neurol 2006;19:84-90.

10. Taub E, Miller NE, Novack TA, et al. Technique to improve chronic motor deficit after stroke. Arch Phys Med Rehabil 1993; 74:347-354.

11. Taub E, Uswatte G, Pidikiti R. Constraint-induced movement therapy: a new family of techniques with broad application to physical rehabilitation-a clinical review. J Rehabil Res Dev 1999;36:237-251.

12. Taub E, Uswatte G, Elbert T. New treatments in neurorehabili- tation founded on basic research. Nat Rev Neurosci 2002;3:228-236.

13. Luft AR, McCombe-Waller S, Whitall J, et al. Repetitive bilateral arm training and motor cortex activation in chronic stroke: a randomized controlled trial. JAMA 2004;292:1853-1861.

14. Whitall J, McCombe Waller S, Silver KH, Macko RF. Repetitive bilateral arm training with rhythmic auditory cueing improves motor function in chronic hemiparetic stroke. Stroke 2000;31: 2390-2395

15. Dobkin B, Apple D, Barbeau H, et al. Weight-supported treadmill vs. over-ground training for walking after acute incomplete SCI. Neurology 2006;66:484-493.

16. Dobkin B, Apple D, Barbeau H, et al. Methods for a randomized trial of weight-supported treadmill training versus conventional training for walking during inpatient rehabilitation after incomplete traumatic spinal cord injury. Neurorehabil Neural Repair 2003;17:153-167.

17. Sullivan KJ, Knowlton BJ, Dobkin BH. Step training with body weight support: effect of treadmill speed and practice paradigms on poststroke locomotor recovery. Arch Phys Med Rehabil 2002; 83:683-691.

18. Hesse S, Schmidt H, Werner C, Bardeleben A. Upper and lower extremity robotic devices for rehabilitation and for studying motor control. Curr Opin Neurol 2003;16:705-710.

19. Hesse S, Schulte-Tigges G, Konrad, M, Bardeleben A, Werne C. Robot-assisted arm trainer for the passive and active practice of bilateral forearm and wrist movements in hemiparetic subjects. Arch Phys Med Rehabil 2003;84:915-920.

20. Lum PS, Burgar CG, Shor PC, Majmundar M, van der Loos M. Robot-assisted movement training compared with conventional therapy techniques for the rehabilitation of upper-limb motor function after stroke. Arch Phys Med Rehabil 2002;83:952-959.

21. Patton JL, Stoykov ME, Kovic M, Mussa-Ivaldi FA. Evaluation of robotic training forces that either enhance or reduce error in chronic hemiparetic stroke survivors. Exp Brain Res 2006;168: 368-383.

22. Stein J, Krebs HI, Frontera WR, Fasoli SE, Hughes R, Hogan N. Comparison of two techniques of robot-aided upper limb exercise training after stroke. Am J Phys Med Rehabil 2004;83:720-728.

23. Volpe BT, Ferraro M, Lynch D, et al. Robotics and other devices in the treatment of patients recovering from stroke. Curr Neurol Neurosci Rep 2005;5:465-470.

24. Volpe BT, Krebs HI, Hogan N. Is robot-aided sensorimotor training in stroke rehabilitation a realistic option? Curr Opin Neurol 2001;14:745-752.

25. Deutsch JE, Merians AS, Adamovich S, Poizner H, Burdea GC. Development and application of virtual reality technology to improve hand use and gait of individuals post-stroke. Restor Neurol Neurosci 2004;22:371-386.

26. Fung J, Richards CL, Malouin F, McFadyen BJ, Lamontagne A. A treadmill and motion coupled virtual reality system for gait training post-stroke. Cyberpsychol Behav 2006;9:157-162.

27. Holden MK. Virtual environments for motor rehabilitation: review. Cyberpsychol Behav 2005;8:187-211; discussion 212-219.

28. Katz N, Ring H, Naveh Y, Kizony R, Feintuch U, Weiss PL. Interactive virtual environment training for safe street crossing of right hemisphere stroke patients with unilateral spatial neglect. Disabil Rehabil 2005;27:1235-1243.

29. Kuttuva M, Boian R, Merians A, et al. The Rutgers Arm, a rehabilitation system in virtual reality: a pilot study. Cyberpsychol Behav 2006;9:148-152.

30. Viau A, Feldman AG, McFadyen BJ, Levin MF. Reaching in reality and virtual reality: a comparison of movement kinematics in healthy subjects and in adults with hemiparesis. J Neuroengineering Rehabil [serial online] 2004;1:11. Available at: http:// www.jneuroengrehab.com/Access Date: December 14, 2004.

31. Ward NS, Cohen LG. Mechanisms underlying recovery of motor function after stroke. Arch Neurol 2004. 61:1844-1848.

32. Calautti C, Baron J-C. Functional neuroimaging studies of motor recovery after stroke in adults: a review. Stroke 2003;34:15531566.

33. Wall PD, Egger M. Formation of new connexions in adult rat brains after partial deafferentation. Nature 1971;232:542-545. 
34. Raisman G, Field PM. A quantitative investigation of the development of collateral reinnervation after partial deafferentation of the septal nuclei. Brain Res 1973;50:241-264.

35. Chklovskii DB, Mel BW, Svoboda K. Cortical rewiring and information storage. Nature 2004;431:782-788.

36. Bliss TV, Gardner-Medwin AR. Long-lasting potentiation of synaptic transmission in the dentate area of the unanaesthetized rabbit following stimulation of the perforant path. J Physiol 1973; 232:357-374.

37. Bliss TV, Lomo T. Long-lasting potentiation of synaptic transmission in the dentate area of the anaesthetized rabbit following stimulation of the perforant path. J Physiol 1973;232:331-356.

38. Byrnes ML, Thickbroom GW, Phillips BA, Mastaglia FL. Longterm changes in motor cortical organization after recovery from subcortical stroke. Brain Res 2001;889:278-287.

39. Finger S, Koehler PJ, Jagella C. The Monakow concept of diaschisis: origins and perspectives. Arch Neurol 2004;61:283-288.

40. Flint AC, Naley MC, Wright CB. Ataxic hemiparesis from strategic frontal white matter infarction with crossed cerebellar diaschisis. Stroke [serial online] 2006;37:e1-2. Epub 2005 Nov. 23. Available at: stroke.ahajournals.org; unedited author manuscript available at http://www.pubmedcentral.gov.

41. Infeld B, Davis SM, Lichtenstein M, Mitchell PJ, Hopper JL. Crossed cerebellar diaschisis and brain recovery after stroke. Stroke 1995;26:90-95.

42. Miura H, Nagata K, Hirata Y, Satoh Y, Watahiki Y, Hatazawa J. Evolution of crossed cerebellar diaschisis in middle cerebral artery infarction. J Neuroimaging 1994;4:91-96.

43. Pantano P, Lenzi GL, Guidetti B, et al. Crossed cerebellar diaschisis in patients with cerebral ischemia assessed by SPECT and ${ }^{123}$ I-HIPDM. Eur Neurol 1987;27:142-148.

44. Sobesky J, Thiel A, Ghaemi M, et al. Crossed cerebellar diaschisis in acute human stroke: a PET study of serial changes and response to supratentorial reperfusion. J Cereb Blood Flow Metab 2005;25:1685-1691.

45. Cappa SF, Perani D, Grassi F, et al. A PET follow-up study of recovery after stroke in acute aphasics. Brain Lang 1997;56:5567.

46. Rizzo M, Robin DA. Bilateral effects of unilateral visual cortex lesions in human. Brain 1996;119:951-963.

47. Seitz RJ, Azari NP, Knorr U, Binkofski F, Herzog H, Freund HJ. The role of diaschisis in stroke recovery. Stroke 1999;30:18441850.

48. Kwakkel G, Kollen B, Lindeman E. Understanding the pattern of functional recovery after stroke: facts and theories. Restor Neurol Neurosci 2004;22:281-299.

49. Feeney DM. From laboratory to clinic: noradrenergic enhancement of physical therapy for stroke or trauma patients. Adv Neurol 1997;73:383-394.

50. Martinsson L, Eksborg S. Drugs for stroke recovery: the example of amphetamines. Drugs Aging 2004;21:67-79.

51. Nudo RJ, Wise BM, SiFuentes F, Milliken GW. Neural substrates for the effects of rehabilitative training on motor recovery after ischemic infarct. Science 1996;272:1791-1794.

52. Nudo RJ, Milliken GW. Reorganization of movement representations in primary motor cortex following focal ischemic infarcts in adult squirrel monkeys. J Neurophysiol 1996;75:2144-2149.

53. Jaillard A, Martin CD, Garambois K, Lebas JF, Hommel M. Vicarious function within the human primary motor cortex? A longitudinal fMRI stroke study. Brain 2005;128:1122-1138.

54. Cramer SC, Nelles G, Benson RR, et al. A functional MRI study of subjects recovered from hemiparetic stroke. Stroke 1997;28: 2518-2527.

55. Cramer SC, Shah R, Juranek J, Crafton KR, Le V. Activity in the peri-infarct rim in relation to recovery from stroke. Stroke 2006; 37:111-115.

56. Loubinoux I, Carel C, Pariente J, et al. Correlation between cerebral reorganization and motor recovery after subcortical infarcts. Neuroimage 2003;20:2166-2180.

57. Werhahn KJ, Conforto AB, Kadom N, Hallett M, Cohen LG. Contribution of the ipsilateral motor cortex to recovery after chronic stroke. Ann Neurol 2003;54:464-472.
58. Fridman EA, Hanakawa T, Chung M, Hummel F, Leiguarda RC, Cohen LG. Reorganization of the human ipsilesional premotor cortex after stroke. Brain 2004;127:747-758.

59. Calautti C, Leroy F, Guincestre JY, Marie RM, Baron JC. Sequential activation brain mapping after subcortical stroke: changes in hemispheric balance and recovery. Neuroreport 2001; 12:3883-3886.

60. Marshall RS, Perera GM, Lazar RM, Krakauer JW, Constantine RC, DeLaPaz RL. Evolution of cortical activation during recovery from corticospinal tract infarction. Stroke 2000;31:656-661.

61. Feydy A, Carlier R, Roby-Brami A, et al. Longitudinal study of motor recovery after stroke: recruitment and focusing of brain activation. Stroke 2002;33:1610-1617.

62. Johansen-Berg H, Rushworth MF, Bogdanovic MD, Kischka U, Wimalaratna S, Matthews PM. The role of ipsilateral premotor cortex in hand movement after stroke. Proc Natl Acad Sci U S A 2002;99:14518-14523.

63. Lotze M, Markert J, Sauseng P, Hoppe J, Plewnia C, Gerloff C. The role of multiple contralesional motor areas for complex hand movements after internal capsular lesion. J Neurosci 2006;26: 6096-6102.

64. Rossini PM, Calautti C, Pauri F, Baron JC. Post-stroke plastic reorganisation in the adult brain. Lancet Neurol 2003;2:493-502.

65. Murase N, Duque J, Mazzocchio R, Cohen LG. Influence of interhemispheric interactions on motor function in chronic stroke. Ann Neurol 2004;55:400-409.

66. Meyer BU, Roricht S, Grafin von Einsiedel H, Kruggel F, Weindl A. Inhibitory and excitatory interhemispheric transfers between motor cortical areas in normal humans and patients with abnormalities of the corpus callosum. Brain 1995;118:429-440.

67. Chen R. Interactions between inhibitory and excitatory circuits in the human motor cortex. Exp Brain Res 2004;154:1-10.

68. Liepert J, Hallett M, Samii A, et al. Motor cortex excitability in patients with cerebellar degeneration. Clin Neurophysiol 2000; 111:1157-1164.

69. Cicinelli P, Pasqualetti P, Zaccagnini M, Traversa R, Oliveri M, Rossini PM. Interhemispheric asymmetries of motor cortex excitability in the postacute stroke stage: a paired-pulse transcranial magnetic stimulation study. Stroke 2003;34:2653-2658.

70. Manganotti P, Patuzzo S, Cortese F, Palermo A, Smania N, Fiaschi A. Motor disinhibition in affected and unaffected hemisphere in the early period of recovery after stroke. Clin Neurophysiol 2002;113:936-943.

71. Kapur N. Paradoxical functional facilitation in brain-behaviour research: a critical review. Brain 1996;119:1775-1790.

72. Finger S, Beyer T, Koehler PJ. Dr. Otto Soltmann (1876) on development of the motor cortex and recovery after its removal in infancy. Brain Res Bull 2000;3:133-140.

73. Nudo RJ, Milliken GW, Jenkins WM, Merzenich MM. Usedependent alterations of movement representations in primary motor cortex of adult squirrel monkeys. J Neurosci 1996;16:785807.

74. Xerri C, Merzenich MM, Peterson BE, Jenkins W. Plasticity of primary somatosensory cortex paralleling sensorimotor skill recovery from stroke in adult monkeys. J Neurophysiol 1998;79: 2119-2148.

75. Brown JA, Lutsep HL, Weinand M, Cramer SC. Motor cortex stimulation for the enhancement of recovery from stroke: a prospective, multicenter safety study. Neurosurgery 2006;58:464473.

76. Teskey GC, Flynn C, Goertzen CD, Monfils MH, Young NA. Cortical stimulation improves skilled forelimb use following a focal ischemic infarct in the rat. Neurol Res 2003;25:794-800.

77. Hallett M. Transcranial magnetic stimulation and the human brain. Nature 2000;406:147-150.

78. Siebner HR, Rothwell J. Transcranial magnetic stimulation: new insights into representational cortical plasticity. Exp Brain Res 2003;148:1-16.

79. Paulus W. Transcranial direct current stimulation (tDCS). Suppl Clin Neurophysiol 2003;56:249-254.

80. Wassermann EM, Grafman J. Recharging cognition with DC brain polarization. Trends Cogn Sci 2005;9:503-505. 
81. Nitsche MA, Liebetanz D, Antal A, Lang N, Tergau F, Paulus W. Modulation of cortical excitability by weak direct current stimulation: technical, safety and functional aspects. Suppl Clin Neurophysiol 2003;56:255-276.

82. Gandiga PC, Hummel FC, Cohen LG. Transcranial DC stimulation (tDCS): a tool for double-blind sham-controlled clinical studies in brain stimulation. Clin Neurophysiol 2006;117:845-850.

83. Wassermann EM. Risk and safety of repetitive transcranial magnetic stimulation: report and suggested guidelines from the International Workshop on the Safety of Repetitive Transcranial Magnetic Stimulation, June 5-7, 1996. Electroencephalogr Clin Neurophysiol 1998;108:1-16.

84. Iyer MB, Mattu U, Grafman J, Lomarev M, Sato S, Wassermann EM. Safety and cognitive effect of frontal DC brain polarization in healthy individuals. Neurology 2005;64:872-875.

85. Lang N, Siebner R, Ward NS, et al. How does transcranial DC stimulation of the primary motor cortex alter regional neuronal activity in the human brain? Eur J Neurosci 2005;22:495-504.

86. Bestmann S, Baudewig J, Siebner HR, Rothwell JC, Frahm J. Functional MRI of the immediate impact of transcranial magnetic stimulation on cortical and subcortical motor circuits. Eur J Neurosci 2004;19:1950-1962.

87. Wagner T, Fregni F, Eden U, et al. Transcranial magnetic stimulation and stroke: a computer-based human model study. Neuroimage 2006;30:857-870.

88. Kobayashi M, Hutchinson S, Theoret H, Schlaug G, PascualLeone A. Repetitive TMS of the motor cortex improves ipsilateral sequential simple finger movements. Neurology 2004;62:91-98.

89. Kim YH, You SH, Ko MH, et al. Repetitive transcranial magnetic stimulation-induced corticomotor excitability and associated motor skill acquisition in chronic stroke. Stroke 2006.

90. Tegenthoff M, Ragert P, Pleger B, et al. Improvement of tactile discrimination performance and enlargement of cortical somatosensory maps after $5 \mathrm{~Hz}$ rTMS. PLoS Biol [serial online] 2005; 3:e362. Epub 2005 Oct. 18. Available at: http://biology.plosjournals.org.

91. Rosen SC, Stamm JS. Transcortical polarization: facilitation of delayed response performance by monkeys. Exp Neurol 1972;35: 282-289.

92. Elbert T, Rockstroh B, Lutzenberger W, Birbaumer N. The influence of low-level, event-related DC-currents during time estimation in humans. Int J Neurosci 1981;15:103-106.

93. Elbert T, Rockstroh B, Lutzenberger W, Birbaumer N. Influence of low-level transcortical DC-currents on response speed in humans. Int J Neurosci 1981;14:101-114.

94. Nitsche MA, Schauenburg A, Lang N, et al. Facilitation of implicit motor learning by weak transcranial direct current stimulation of the primary motor cortex in the human. J Cogn Neurosci 2003;15:619-626.

95. Fregni F, Boggio PS, Mansur CG, et al. Transcranial direct current stimulation of the unaffected hemisphere in stroke patients. Neuroreport 2005;16:1551-1555.

96. Kincses TZ, Antal A, Nitsche MA, Bartfai O, Paulus W. Facilitation of probabilistic classification learning by transcranial direct current stimulation of the prefrontal cortex in the human. Neuropsychologia 2004;42:113-117.

97. Antal A, Nitsche MA, Kruse W, Kincses TZ, Hoffmann KP, Paulus W. Direct current stimulation over V5 enhances visuomo- tor coordination by improving motion perception in humans. $\mathrm{J}$ Cogn Neurosci 2004;6:521-527.

98. Bear MF, Malenka R. Synaptic plasticity: LTP and LTD. Curr Opin Neurobiol 1994;4:389-399.

99. Woody CD, Gruen E, Birt D. Changes in membrane currents during Pavlovian conditioning of single cortical neurons. Brain Res 1991;539:76-84.

100. Dancause N, Barbay S, Frost SB, et al. Extensive cortical rewiring after brain injury. J Neurosci 2005;25:10167-10179.

101. Hummel F, Celnik P, Giraux P, et al. Effects of non-invasive cortical stimulation on skilled motor function in chronic stroke. Brain 2005;128:490-499.

102. Hummel F, Cohen LG. Improvement of motor function with noninvasive cortical stimulation in a patient with chronic stroke. Neurorehabil Neural Repair 2005;19:14-19.

103. Jebsen RH, Taylor N, Trieschmann RB, Trotter MJ, Howard LA. An objective and standardized test of hand function. Arch Phys Med Rehabil 1969;50:311-319.

104. Kraft GH, Fitts SS, Hammond MC. Techniques to improve function of the arm and hand in chronic hemiplegia. Arch Phys Med Rehabil 1992;73:220-227.

105. Alon G, Sunnerhagen KS, Geurts AC, Ohry A. A home-based, self-administered stimulation program to improve selected hand functions of chronic stroke. Neurorehabilitation 2003;18:215225.

106. Wu CW, Seo HJ, Cohen LG. Influence of electric somatosensory stimulation on paretic-hand function in chronic stroke. Arch Phys Med Rehabil 2006;87:351-357.

107. Khedr EM, Ahmed MA, Fathy N, Rothwell JC. Therapeutic trial of repetitive transcranial magnetic stimulation after acute ischemic stroke. Neurology 2005;65:466-468.

108. Swinnen SP. Intermanual coordination: from behavioural principles to neural-network interactions. Nat Rev Neurosci 2002;3: $348-359$.

109. Plewnia C, Lotze M, Gerloff C. Disinhibition of the contralateral motor cortex by low-frequency rTMS. Neuroreport 2003;14:609612.

110. Schambra HM, Sawaki L, Cohen LG. Modulation of excitability of human motor cortex (M1) by $1 \mathrm{~Hz}$ transcranial magnetic stimulation of the contralateral M1. Clin Neurophysiol 2003;114: $130-133$.

111. Kobayashi M, Hutchinson S, Schlaug G, Pascual-Leone A. Ipsilateral motor cortex activation on functional magnetic resonance imaging during unilateral hand movements is related to interhemispheric interactions. Neuroimage 2003;20:2259-2270.

112. Takeuchi N, Chuma T, Matsuo Y, Watanabe I, Ikoma K. Repetitive transcranial magnetic stimulation of contralesional primary motor cortex improves hand function after stroke. Stroke 2005; 36:2681-2686.

113. Mansur CG, Fregni F, Boggio PS, et al. A sham stimulationcontrolled trial of rTMS of the unaffected hemisphere in stroke patients. Neurology 2005;64:1802-1804.

114. Naeser MA, Martin PI, Nicholas M, et al. Improved picture naming in chronic aphasia after TMS to part of right Broca's area: an open-protocol study. Brain Lang 2005;93:95-105.

115. Naeser MA, Martin PI, Nicholas M, et al. Improved naming after TMS treatments in a chronic, global aphasia patient-case report. Neurocase 2005;11:182-193. 